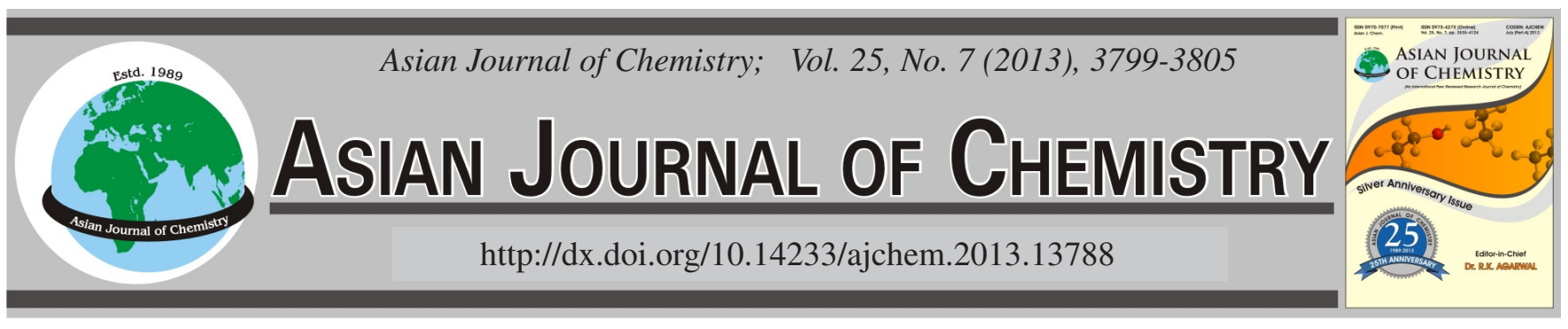

\title{
Structural Identification of Chemical Constituents from Scutellaria baicalensis by HPLC-ESI-MS/MS and NMR Spectroscopy
}

\author{
Wenhui Lin ${ }^{1}$, ShuAng Liv ${ }^{2,3}$ and Bin Wu ${ }^{3, *}$ \\ ${ }^{1}$ School of Chemical and Environmental Engineering, Shanghai Institute of Technology, Shanghai 201418, P.R. China \\ ${ }^{2}$ Haerbin University of Commerce, Haerbin 150076, P.R. China \\ ${ }^{3}$ Shanghai Institute of Materia Medica, Chinese Academy of Sciences, 501 Haike Rd, Zhangjiang, Pudong 201203, Shanghai, P.R. China \\ *Corresponding author: Email: wubin1031@hotmail.com; 893898379@qq.com; linwu1217@hotmail.com
}

\begin{abstract}
Scutellariae Radix, the dried roots of Scutellaria baicalensis Georgi, which is one of the famous herb widely used in the treatment of pyrexia, jaundice and hypertension. To directly identify the compounds of the extract of the S. baicalensis was performed using HPLCESI-MS/MS. Chromatographic (analytical and preparative HPLC), chemical (hydrolysis) and spectroscopic (UV, ${ }^{1} \mathrm{H}$ and ${ }^{13} \mathrm{C}$ NMR, MS) techniques were applied for separation, isolation, purification and identification of new compounds. A systematic phytochemical investigantion of $S$. baicalensis led to the isolation of 7 new and 40 known chemical constituents. By HPLC-ESI-MS/MS and NMR spectral analyses, the new compounds were identified as 7-hydroxy-3,5,8-trimethoxy-3',4'-methylenedioxy flavone-7-O- $\beta$ - glucopyranoside (1), 7-hydroxy-5,6,8-trimethoxy-3',4'-methylenedioxy flavone-7- $O$ - $\beta$-glucopyranoside (2), patuletin-7- $\beta$-glucuronide (3), pinobankasin6-C-glucopyranosyl-8-C-arabinopyranoside (4), chrysin-3-C- $\alpha$-arabinopyranosyl-8-C- $\beta$-glucopyranoside (11), 6-sulfooxy-chrysin-7glucoside (12) and 5-hydoxy-6,7,4'-trimethoxy-flavanone 5-sulfate (24).
\end{abstract}

Key Words: Scutellaria baicalensis; Structural elucidation, Chemical constituents, HPLC-MS/MS, NMR.

\section{INTRODUCTION}

Huangqin (Radix scutellariae) is an important traditional Chinese medicine prepared from the roots of Scutellaria baicalensis Georgi (Labiatae family). It is widely used in traditional Chinese medicine to treat inflammation, pyrexia, jaundice, hepatitis and hypertension ${ }^{1}$. In the clinic, it is widely applied to cure pneumonia, hypertension, jaundice, dysentery and intestinal catarrh, pyogenic infection, etc. ${ }^{2}$. It acts as a key ingredient in a number of formulae such as Shuanghuanglian oral liquid, Huangqin compound recipes granule, Yinhuang table, etc. Flavonoids and their derivatives are the main components of the $S$. baicalensis. Including baicalin, baicalein, oroxylin A, wogonoside, wogonin, apigenin and scutellarein, etc. ${ }^{3}$.

This paper describes 7 new and 38 known chemical consitituents, including 2 flavone sulfates representing the first report of natural flavone sulfates from this genus and 5 novel flavone glycosides, from the aerial parts of this plant. The molecular structures of these new compounds were elucidated based on ${ }^{1} \mathrm{D}$ and ${ }^{2} \mathrm{D}$ NMR spectra and comparisons with literature data.

\section{EXPERIMENTAL}

Optical rotations were measured using a Perkin-Elmer 241 MC polarimeter. IR spectra were recorded using a PerkinElmer 577 spectrometer. UV spectra were obtained on a Shimadzu UV-2401PC spectrophotometer. HR-TOF-MS was performed using a Waters Q-TOF Ultima. NMR experiments were run on a Varian Mercury-400 spectrometer (USA). Chemical shifts are given on a $\delta$ (ppm) scale using TMS as an internal standard. HPLC was performed using Davisil ${ }^{\circledR}$ ODS (35-60 $\mu \mathrm{m}, 60 \AA$, Grace) on a BUCHI C-615 pump (UVdetector C-635 and C-660 fraction collector, BUCHI, Switzerland). Semi-preparative HPLC was carried out using EasySep TM-1010 instrument equipped with a Daisogel $\mathrm{C}_{18}$ column $(12 \mu \mathrm{m}, 20 \mathrm{~mm} \times 250 \mathrm{~mm})$.

HPLC conditions: An Agilent series 1100 HPLC instrument (Waldbronn, Germany) equipped with a quaternary pump, a UV detector and a column compatment was used for analyses. The samples were separated on a Apollo $\mathrm{C}_{18}$ column $(5 \mu \mathrm{m}, 4.6 \times 250 \mathrm{~mm}$, Grace), including an Easy Guard Kit $\mathrm{C}_{18}(4 \times 2 \mathrm{~mm}$, Dikma $)$ guard column. A gradient elution of $0.5 \%$ aqueous formic acid (A) and acetonitrile (B) was used 

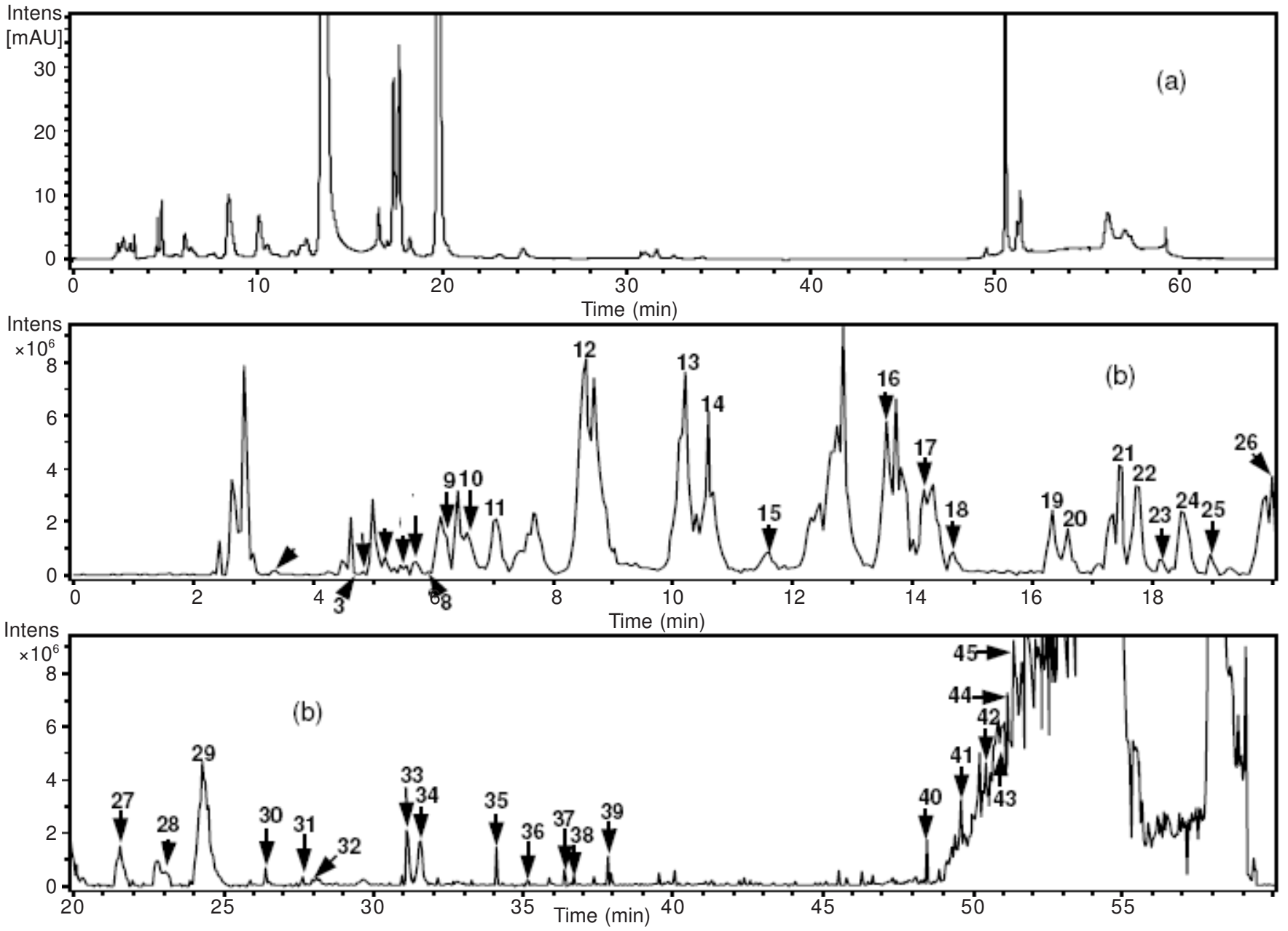

Fig. 1. A representative HPLC-ESI-MSn analysis of the extract of S. baicalensis. (a) HPLC-UV chromatogram at $280 \mathrm{~nm}$ (b) LC-negative ion ESI-MS total ion current (TIC) profile

as follows: $20 \% \mathrm{~B}$ in the first $10 \mathrm{~min}, 20 \%-25 \% \mathrm{~B}$ at $10-11$ min, then B held at $25 \%$ for 14 min, linearly gradient to $35 \%$ $\mathrm{B}$ at $26 \mathrm{~min}$ and hold for $15 \mathrm{~min}, 35 \%-55 \% \mathrm{~B}$ at $41-45 \mathrm{~min}$, linearly gradient to $100 \% \mathrm{~B}$ at $50 \mathrm{~min}$ and hold for $5 \mathrm{~min}$. The column was maintained at $25^{\circ} \mathrm{C}$. Detection wavelengths were set at $280 \mathrm{~nm}$. The flow rate was $0.8 \mathrm{~mL} / \mathrm{min}$ (Fig. 1).

Mass spectrometric conditions: HPLC-MS experiments were performed with an Agilent 1100 series LC/MSD Trap mass spectrometer was connected to an Agilent 1100 HPLC instrument via an ESI source. The LC/MSD Trap software (5.3 version) was applied for system operation and data collection. The mass spectra were recorded in negative mode, drying gas flow rate $10 \mathrm{~L} / \mathrm{min}$, drying gas temperature $350{ }^{\circ} \mathrm{C}$, nebulizer 35 psi., capillary voltage $3500 \mathrm{~V}$, mass range 50$1500 \mathrm{~m} / \mathrm{z}$.

The roots of Scutellaria baicalensis Georgi were collected from Xi'an, ShanXi province, China and authenticated by Professor Shen Jingui of Shanghai Institute of Materia Medica, Chinese Academy of Sciences. A voucher specimen (SIMM-HCG-HQ0905) was deposited in the Herbarium of Shanghai Institute of Materia Medica, Chinese Academy of Sciences.

Extraction and isolation: The roots of S. baicalensis (5 $\mathrm{Kg}$ ) were extracted twice with $95 \%$ ethanol at room temperature. The EtOH extracts were combined and evaporated under reduced pressure to give a residue $(677 \mathrm{~g})$. A protion of the extract (384 g) was suspended in distilled water and chromatographed using medium-pressure ODS column chromatography (column dimensions $100 \mathrm{~mm} \times 460 \mathrm{~mm}$ ) eluting followed by increasing concentrations of $\mathrm{MeOH}$ in $\mathrm{H}_{2} \mathrm{O}$ (10, 20, 30, 50, 80 and $100 \% \mathrm{MeOH}$; fraction volume $1200 \mathrm{~mL}$ ). Give 48 fractions $(355 \mathrm{~g})$, which were combined into seven main groups (A-G). The fraction A (33.8 g) eluted with $10 \%$ $\mathrm{MeOH}$ was rechromatographed using semi-preparative HPLC to give compound 1 (5.4 $\mathrm{mg}$ ) and compound 2 (6.0 $\mathrm{mg}$ ). Fraction B (26.4 g) eluted with $13 \% \mathrm{MeOH}$ was repeatedly chromatographed using semi-preparative HPLC to yield compound $\mathbf{3}(4.5 \mathrm{mg})$ and compound $4(5.4 \mathrm{mg})$. The fraction $\mathrm{C}(11.4 \mathrm{~g})$ eluted with $15 \% \mathrm{MeOH}$ was rechromatographed using semi-preparative HPLC to yield compound $\mathbf{1 1}$ (15.7 mg). The fraction D $(93.9 \mathrm{~g})$ eluted with $17 \% \mathrm{MeOH}$ was rechromatographed using semi-preparative HPLC to give compound $12(21.1 \mathrm{mg})$. The fraction F (73.9 g) eluted with $22 \% \mathrm{MeOH}$ was rechromatographed using semi-preparative HPLC to give compound $\mathbf{2 4}(6.8 \mathrm{mg})$.

\section{Experimental data for the new flavonoid glycosides}

7-Hydroxy-3,5,8-trimethoxy-3',4' -methylenedioxy flavone-7-O- $\boldsymbol{\beta}$-glucopyranoside (1): Pale yellow needles crystals; m.p.: $284-285{ }^{\circ} \mathrm{C}$; UV $(\mathrm{MeOH}) \lambda_{\max }(\log \varepsilon): 256$ (4.21), 273 (4.23), 350 (4.23); IR (KBr, $\left.\nu_{\max }, \mathrm{cm}^{-1}\right): 3100(\mathrm{OH})$, $1620(\mathrm{C}=\mathrm{O}), 1605,1590,1495$; ESI-MS [M-H]- $m / z, 533$; HR- 
ESI-MS [M-H]- $m / z, 533.1274$ (calcd. for $\mathrm{C}_{25} \mathrm{H}_{26} \mathrm{O}_{13}$, 533.1295); ${ }^{1} \mathrm{H}$ NMR (DMSO- $\left.d_{6}, 400 \mathrm{MHz}\right) \delta 7.69(1 \mathrm{H}, \mathrm{dd}$, $\left.J=8.5,2.0 \mathrm{~Hz}, \mathrm{H}-6^{\prime}\right), 7.54\left(1 \mathrm{H}, \mathrm{d}, J=2.0 \mathrm{~Hz}, \mathrm{H}-2^{\prime}\right), 7.13(1 \mathrm{H}$, d, $J=8.5 \mathrm{~Hz}, \mathrm{H}-5$ '), 6.41 (1H, s, H-6), $6.13\left(2 \mathrm{H}, \mathrm{s},-\mathrm{O}-\mathrm{CH}_{2}-\mathrm{O}-\right.$ ), $5.11(1 \mathrm{H}, \mathrm{d}, J=7.1 \mathrm{~Hz}, \mathrm{Glc}-1), 3.90(1 \mathrm{H}, \mathrm{m}, \mathrm{Glc}-6), 3.81$ $\left(3 \mathrm{H}, \mathrm{s}, 5-\mathrm{OCH}_{3}\right), 3.75\left(3 \mathrm{H}, \mathrm{s}, 8-\mathrm{OCH}_{3}\right), 3.72\left(3 \mathrm{H}, \mathrm{s}, 3-\mathrm{OCH}_{3}\right)$, 3.71 (1H, m, Glc-6), 3.46 (1H, m, Glc-5), 3.38 (1H, m, Glc3), $3.35(1 \mathrm{H}, \mathrm{m}, \mathrm{Glc}-4), 3.21(1 \mathrm{H}, \mathrm{m}, \mathrm{Glc}-2) ;{ }^{13} \mathrm{C} \mathrm{NMR}$ (DMSO- $\left.d_{6}, 100 \mathrm{MHz}\right) \delta 174.8(\mathrm{C}-4), 157.1(\mathrm{C}-5), 155.4(\mathrm{C}-$ 7), 152.0 (C-2), 151.3 (C-3'), 149.2 (C-9), 147.5 (C-4'), 140.9 (C-3), 131.0 (C-8), 124.8 (C-1'), 119.0 (C-6'), 109.3 (C-5'), 109.0 (C-10), 108.1 (C-2'), 103.1 (-O-CH $2-\mathrm{O}-), 99.5$ (Glc-1), 94.2 (C-6), 77.0 (Glc-5), 76.3 (Glc-3), 73.1 (Glc-2), 69.6 (Glc4), $61.2\left(8-\mathrm{OCH}_{3}\right), 60.7$ (Glc-6), $59.7\left(3-\mathrm{OCH}_{3}\right), 56.4$ (5$\mathrm{OCH}_{3}$ ); ESI-MS/MS data (Table-1).

7-Hydroxy-5,6,8-trimethoxy-3',4'-methylenedioxy flavone-7-O- $\boldsymbol{\beta}$-glucopyranoside (2): Pale yellow needles; UV $(\mathrm{MeOH}) \lambda_{\max }(\log \varepsilon): 257,350 \mathrm{~nm}$. IR $\left(\mathrm{KBr}, v_{\max }, \mathrm{cm}^{-1}\right): 3100$ $(\mathrm{OH}), 1620(\mathrm{C}=\mathrm{O}), 1605,1590,1495$; ESI-MS [M-H]- $m / z$, 533; HR-ESI-MS [M-H]- $m / z, 533.1275$ (calcd. for $\mathrm{C}_{25} \mathrm{H}_{26} \mathrm{O}_{13}$, 533.1295); ${ }^{1} \mathrm{H}$ NMR (DMSO- $\left.d_{6}, 400 \mathrm{MHz}\right) \delta 7.69(1 \mathrm{H}, \mathrm{dd}$, $\left.J=8.8,2.3 \mathrm{~Hz}, \mathrm{H}-6^{\prime}\right), 7.55$ (1H, d, $\left.J=2.3 \mathrm{~Hz}, \mathrm{H}-2 '\right), 7.14(1 \mathrm{H}$, d, $\left.J=8.8 \mathrm{~Hz}, \mathrm{H}-5^{\prime}\right), 6.63(1 \mathrm{H}, \mathrm{s}, \mathrm{H}-3), 6.11\left(2 \mathrm{H}, \mathrm{s},-\mathrm{O}-\mathrm{CH}_{2}-\mathrm{O}-\right.$ ), $5.08(1 \mathrm{H}, \mathrm{d}, J=7.3 \mathrm{~Hz}, \mathrm{Glc}-1), 3.92\left(3 \mathrm{H}, \mathrm{s}, 5-\mathrm{OCH}_{3}\right), 3.90$ $(1 \mathrm{H}, \mathrm{m}, \mathrm{Glc}-6), 3.89\left(3 \mathrm{H}, \mathrm{s}, 8-\mathrm{OCH}_{3}\right), 3.85\left(3 \mathrm{H}, \mathrm{s}, 6-\mathrm{OCH}_{3}\right)$, $3.71(1 \mathrm{H}, \mathrm{m}, \mathrm{Glc}-6), 3.46$ (1H, m, Glc-5), 3.39 (1H, m, Glc3), $3.35(1 \mathrm{H}, \mathrm{m}, \mathrm{Glc}-4), 3.21(1 \mathrm{H}, \mathrm{m}, \mathrm{Glc}-2) ;{ }^{13} \mathrm{C} \mathrm{NMR}$ $\left(\mathrm{DMSO}-d_{6}, 100 \mathrm{MHz}\right) \delta 175.1(\mathrm{C}-4), 160.6(\mathrm{C}-2), 151.5(\mathrm{C}-$ 3'), 150.3 (C-6), 149.1 (C-8), 147.8 (C-4'), 147.6 (C-9), 144.0 (C-5), 137.1 (C-7), 124.3 (C-1'), 119.3 (C-6'), 110.1 (C-5'), 109.5 (C-10), 109.0 (C-2'), 106.3 (C-3), 103.2 (-O-CH2-O-), 99.9 (Glc-1), 77.1 (Glc-5), 76.4 (Glc-3), 73.1 (Glc-2), 69.5 (Glc-4), $61.5\left(5-\mathrm{OCH}_{3}\right), 61.2\left(6-\mathrm{OCH}_{3}\right), 61.0\left(8-\mathrm{OCH}_{3}, \mathrm{Glc}-\right.$ $6)$, ESI-MS/MS data (Table-1).

Patuletin-7- $\beta$-glucuronide (3): Pale yellow crystals; UV $(\mathrm{MeOH}) \lambda_{\max }(\log \varepsilon): 255,267 \mathrm{sh}, 336 \mathrm{~nm} ; \mathrm{IR}\left(\mathrm{KBr}, v_{\max }, \mathrm{cm}^{-1}\right)$ : 3327, 1654, 1610, 1504, 1457, 881, 805; ESI-MS [M-H]$m / z, 507$; HR-ESI-MS [M-H]- $m / z, 507.0760$ (calcd. for $\left.\mathrm{C}_{22} \mathrm{H}_{20} \mathrm{O}_{14}, 507.0775\right) ;{ }^{1} \mathrm{H}$ NMR (DMSO- $\left.d_{6}, 400 \mathrm{MHz}\right) \delta 12.31$ $(1 \mathrm{H}, \mathrm{s}, 5-\mathrm{OH}), 9.92\left(1 \mathrm{H}, \mathrm{s}, 3{ }^{\prime}-\mathrm{OH}\right), 9.80(1 \mathrm{H}, \mathrm{s}, 3-\mathrm{OH}), 9.40$ (4'-OH), 7.67 (1H, d, $J=2.1 \mathrm{~Hz}, \mathrm{H}-2$ ') $7.56(1 \mathrm{H}, \mathrm{dd}, J=7.8$, $2.1 \mathrm{~Hz}, \mathrm{H}-6 '), 6.89$ (1H, d, J=7.8 Hz, H-5'), 6.51 (1H, s, H-8), $5.17(1 \mathrm{H}, \mathrm{d}, J=7.4 \mathrm{~Hz}, \mathrm{GlcA}-1), 4.15(1 \mathrm{H}, \mathrm{d}, J=9.5 \mathrm{~Hz}$, GlcA-5), $3.81\left(3 \mathrm{H}, \mathrm{s}, 6-\mathrm{OCH}_{3}\right), 3.67(1 \mathrm{H}, \mathrm{t}, J=9.5 \mathrm{~Hz}, \mathrm{GlcA}-$ 4), 3.59 (2H, m, GlcA-2, GlcA-3); ${ }^{13} \mathrm{C}$ NMR (DMSO- $d_{6}, 100$ MHz) $\delta 176.3$ (C-4), 169.9 (GlcA-6), 157.3 (C-7), 151.5 (C9), 151.4 (C-5), 147.5 (C-4'), 146.5 (C-2), 145.3 (C-3'), 135.1 (C-3), 130.9 (C-6), 122.1 (C-1'), 119.8 (C-6'), 115.5 (C-5'), 114.7 (C-2'), 103.8 (C-10), 101.2 (GlcA-1), 94.5 (C-8), 75.8 (GlcA-5), 75.3 (GlcA-3), 72.8 (GlcA-2), 71.2 (GlcA-4), 59.9 $\left(6-\mathrm{OCH}_{3}\right) ; \mathrm{ESI}-\mathrm{MS} / \mathrm{MS}$ data (Table-1).

Pinobankasin-6-C-glucopyranosyl-8-C-arabinopyranoside (4): Colourless needles; m.p.: $164-166^{\circ} \mathrm{C} ;[\alpha]^{25}=$ 10.1 (c 0.5, MeOH). UV (MeOH) $\lambda_{\max }(\log \varepsilon): 380,315 \mathrm{~nm}$; IR $\left(\mathrm{KBr}, \mathrm{v}_{\max }, \mathrm{cm}^{-1}\right): 3460,3120,1650,1620,1480,1285$, 1178; ESI-MS [M-H]- $m / z, 565$; HR-ESI-MS [M-H]- $m / z$, 565.1563 (calcd. for $\left.\mathrm{C}_{26} \mathrm{H}_{30} \mathrm{O}_{14}, 565.1558\right) ;{ }^{1} \mathrm{H} \mathrm{NMR}\left(\mathrm{CD}_{3} \mathrm{OD}\right.$, $400 \mathrm{MHz}$ ) 7.49 (2H, m, H-2', H-6'), 7.27 (1H, m, H-4'), 7.25
(2H, m, H-3', H-5'), 5.07 (1H, d, J=12.8 Hz, H-3), 4.51 (1H, $\mathrm{d}, J=12.8 \mathrm{~Hz}, \mathrm{H}-2), 4.80(1 \mathrm{H}, \mathrm{d}, J=9.6 \mathrm{~Hz}, \mathrm{Glc}-1), 4.64$ $(1 \mathrm{H}, \mathrm{d}, J=9.0 \mathrm{~Hz}$, Ara-1), $4.01(1 \mathrm{H}, \mathrm{m}$, Ara-2), $3.93(1 \mathrm{H}, \mathrm{m}$, Ara-5), 3.89 (1H, m, Glc-2), 3.88 (1H, m, Ara-4), $3.70(1 \mathrm{H}$, m, Glc-6), 3.69 (1H, m, Ara-5), 3.54 (1H, m, Glc-6), 3.53 (1H, m, Ara-3), 3.28 (3H, m, Glc-3, Glc-4, Glc-5); ${ }^{13} \mathrm{C}$ NMR $\left(\mathrm{CD}_{3} \mathrm{OD}, 100 \mathrm{MHz}\right) \delta 195.6(\mathrm{C}-4), 165.1$ (C-7), $161.5(\mathrm{C}-5)$, 160.5 (C-9), 136.3 (C-1'), 129.2 (C-3', C-5'), 128.7 (C-2', C6'), 127.5 (C-4'), 106.9 (C-6), 105.4 (C-8), 96.8 (C-10), 83.6 (C-2), 77.7 (Glc-3, Glc-5), 75.2 (Ara-3), 74.2 (Ara-1), 73.2 (Glc-1), 72.7 (C-3), 71.1 (Glc-4), 70.8 (Glc-2), 69.6 (Ara-5), 69.1 (Ara-2), 68.6 (Ara-4), 62.2 (Glc-6); ESI-MS/MS data (Table-1).

Chrysin 3-C-a-arabinopyranosyl-8-C- $\boldsymbol{\beta}$-glucopyranoside (11): Pale yellow needles crystals; m.p.: $274-276^{\circ} \mathrm{C}$; UV $(\mathrm{MeOH}) \lambda_{\max }(\log \varepsilon): 269,316 \mathrm{~nm}$; IR $\left(\mathrm{KBr}, v_{\max }, \mathrm{cm}^{-1}\right): 3264$ $(-\mathrm{OH}), 1650$ (-C=O), 1595, 1342, 1216, 845; ESI-MS [M-H]$m / z, 547$; HR-ESI-MS [M-H]- $m / z, 547.1435$ (calcd. for $\left.\mathrm{C}_{26} \mathrm{H}_{28} \mathrm{O}_{13}, 547.1452\right) ;{ }^{1} \mathrm{H}$ NMR (DMSO- $\left.d_{6}, 400 \mathrm{MHz}\right) \delta 12.90$ (1H, s, 5-OH), 10.51 (1H, s, 7-OH), 8.13 (2H, m, H-2', H-6'), 7.70 (1H, m, H-4'), 7.66 (2H, m, H-3', H-5'), 6.27 (1H, s, H6), $4.80(1 \mathrm{H}, \mathrm{d}, J=9.4 \mathrm{~Hz}$, Ara-1), $4.76(1 \mathrm{H}, \mathrm{d}, J=9.8 \mathrm{~Hz}$, Glc-1), 4.00 (2H, m, Glc-2, Ara-2), 3.79 (1H, m, Ara-5), 3.77 (1H, m, Ara-4), 3.74 (1H, m, Glc-6), 3.57 (1H, m, Ara-5), 3.52 (1H, m, Glc-6), 3.42 (1H, m, Ara-3), 3.36 (1H, m, Glc4), $3.30(1 \mathrm{H}, \mathrm{m}, \mathrm{Glc}-3), 3.27(1 \mathrm{H}, \mathrm{m}, \mathrm{Glc}-5) ;{ }^{13} \mathrm{C} \mathrm{NMR}$ (DMSO- $\left.d_{6}, 100 \mathrm{MHz}\right) \delta 178.3$ (C-4), 161.0 (C-5, C-7), 160.1 (C-2), 154.3 (C-9), 131.9 (C-4'), 129.3 (C-1'), 128.7 (C-3', C5'), 126.1 (C-2', C-6'), 101.3 (C-10), 115.3 (C-3), 105.1 (C8), 95.8 (C-6), 81.5 (Glc-5), 79.1 (Glc-3), 74.6 (Ara-1), 74.5 (Glc-1), 73.9 (Ara-3), 71.5 (Glc-2), 70.8 (Glc-4), 70.1 (Ara5), 68.9 (Ara-2), 68.4 (Ara-4), 60.9 (Glc-6); ESI-MS/MS data (Table-1).

6-Sulfooxy-chrysin-7-glucoside (12): Pale yellow needles crytals; UV $\lambda_{\max }(\log \varepsilon): 267,313 \mathrm{~nm}$; IR (KBr, $v_{\max }$, $\mathrm{cm}^{-1}$ ): 3348, 1655, 1617, 1510, 1068, 854, 768, 686; ESI-MS [M-H]- $m / z, 511$; HR-ESI-MS [M-H]- $m / z, 511.0562$ (calcd. for $\mathrm{C}_{21} \mathrm{H}_{20} \mathrm{O}_{13} \mathrm{~S}, 511.0547$ ); ${ }^{1} \mathrm{H}$ NMR (DMSO- $\left.d_{6}, 400 \mathrm{MHz}\right) \delta$ 12.63 (1H, s, 5-OH), 8.08 (2H, m, H-2', H-6'), 7.58 (3H, m, H-3', H-4', H-5'), 7.36 (1H, s, H-8), 7.11 (1H, s, H-3), 4.53 $(1 \mathrm{H}, \mathrm{d}, J=6.8 \mathrm{~Hz}, \mathrm{Glc}-1), 3.92(1 \mathrm{H}, \mathrm{m}, \mathrm{Glc}-6), 3.71(1 \mathrm{H}, \mathrm{m}$, Glc-6), 3.45 (1H, m, Glc-5), 3.37 (1H, m, Glc-3), $3.35(1 \mathrm{H}$, m, Glc-4), 3.20 (1H, m, Glc-2); ${ }^{13} \mathrm{C}$ NMR (DMSO- $d_{6}, 100$ MHz) $\delta 182.6$ (C-4), 166.5 (C-7), 164.5 (C-2), 164.2 (C-5), 153.2 (C-9), 132.5 (C-4'), 130.4 (C-1'), 129.3 (C-3', C-5'), 126.7 (C-2', C-6'), 105.7 (C-3, C-10), 101.3 (Glc-1), 94.7 (C6), 94.4 (C-8), 77.0 (Glc-5), 76.2 (Glc-3), 73.5 (Glc-2), 70.0 (Glc-4), 61.0 (Glc-6); ESI-MS/MS data (Table-1).

5-Hydroxy-6,7,4'-trimethoxy flavanone 5-sulfate (24): Pale yellow crytals; m.p.: $164-166^{\circ} \mathrm{C}$; UV (MeOH) $\lambda_{\max }(\log$ $\varepsilon): 225,287,340 \mathrm{~nm}$; IR (KBr, $\left.\nu_{\max }, \mathrm{cm}^{-1}\right)$ : 3400, 2905, 1630, 1565, 1510, 1445, 1270, 1250, 1190, 1155, 1105, 1005, 965, 890 and 830; ESI-MS [M-H]- $m / z, 409$; HR-ESI-MS [M-H]$m / z, 409.0590$ (calcd. for $\mathrm{C}_{18} \mathrm{H}_{18} \mathrm{O}_{9} \mathrm{~S}, 409.0594$ ); ${ }^{1} \mathrm{H}$ NMR (DMSO- $\left.d_{6}, 400 \mathrm{MHz}\right) \delta 7.48(2 \mathrm{H}, \mathrm{d}, J=8.8 \mathrm{~Hz}, \mathrm{H}-2$ ', H-6'), $6.95\left(2 \mathrm{H}, \mathrm{d}, J=8.8 \mathrm{~Hz}, \mathrm{H}-3^{\prime}, \mathrm{H}-5^{\prime}\right), 6.71$ (1H, s, H-8), 5.57 $(1 \mathrm{H}, \mathrm{dd}, J=8.8,3.8 \mathrm{~Hz}, \mathrm{H}-2), 3.85\left(3 \mathrm{H}, \mathrm{s}, 7-\mathrm{OCH}_{3}\right), 3.80$ $\left(3 \mathrm{H}, \mathrm{s}, 6-\mathrm{OCH}_{3}\right), 3.78\left(3 \mathrm{H}, \mathrm{s}, 4{ }^{\prime}-\mathrm{OCH}_{3}\right), 3.35(1 \mathrm{H}, \mathrm{dd}, J=$ 
TABLE-1

CHARACTERIZATION OF COMPOUNDS BY HPLC-ESI-MS/MS FROM S. baicalensis

\begin{tabular}{|c|c|c|c|c|}
\hline No. & $\begin{array}{c}\mathrm{Rt} \\
(\mathrm{min})\end{array}$ & $\mathrm{UV} \lambda_{\max }(\mathrm{nm})$ & ESI-MS/MS m/z (abundance) & Identification results \\
\hline 1 & 3.3 & $256,273,350$ & $\begin{array}{l}\mathrm{MS}^{2}[533]: \text { 516(1.2), 317(8.4), 353(27.3), 341(7.8), 329(0.6), } \\
\text { 313(2.3) }\end{array}$ & $\begin{array}{l}\text { 7-Hydroxy-3,5,8-trimethoxy-3',4'-(methylene- } \\
\text { dioxy) flavone-7-O- } \beta \text {-glucopyranoside }\end{array}$ \\
\hline 2 & 3.4 & 258,350 & $\mathrm{MS}^{2}[533]:$ 516(1.2), 317(8.4), 353(27.3), 341(7.8), 313(2.3) & $\begin{array}{l}\text { 7-Hydroxy-5,6,8-trimethoxy-3', } 4^{\prime} \text {-(methylene- } \\
\text { dioxy) flavone-7-O- } \beta \text {-glucopyranoside }\end{array}$ \\
\hline 3 & 4.8 & $255,267,336$ & $\operatorname{MS}^{2}$ [507]: 491(26.5), 399(99.3), 331(31.1) & Patuletin-7- $\beta$-glucuronide \\
\hline 4 & 4.9 & 280,315 & $\begin{array}{l}\mathrm{MS}^{2}[565]: \text { 547(100.0), 487(50.7), 457(75.9), } 445 \text { (43.9), } \\
\text { 367(86.0), 337(51.5) }\end{array}$ & $\begin{array}{l}\text { Pinobankasin-6-C-glucopyranosyl-8-C- } \\
\text { arabinopyranoside }\end{array}$ \\
\hline 5 & 5.2 & 255,370 & $\mathrm{MS}^{2}[657]: 495(100.0), 333(0.2)$ & 6,8-Dihydroxy quercetin diglucopyranoside \\
\hline 6 & 5.5 & 282,330 & $\operatorname{MS}^{2}[671]: 509(100.0), 389(4.4), 347(50.4), 228(1.4)$ & $\begin{array}{l}3,5,7,8,3^{\prime}, 4^{\prime} \text {-Hexahydroxy-6-methoxy flavone } \\
\text { diglucopyranoside }\end{array}$ \\
\hline 7 & 5.7 & 271,338 & $\begin{array}{l}\mathrm{MS}^{2}[563]: \text { 443(100.0), 545(15.0), 503(27.0), } 486(14.6), \\
473(67.1), 383(33.9), 353(73.1)\end{array}$ & Apigenin-6-C- $\beta$-D-glucoside-8-C-?-L-arabinoside \\
\hline 8 & 5.9 & 271,338 & $\begin{array}{l}\mathrm{MS}^{2}[563]: 443(100.0), 545(28.5), 503(44.4), 485(15.6), \\
473(67.7), 425(26.3), 383(38.7), 353(34.8)\end{array}$ & Apigenin-6-C- $\alpha$-L-arabinoside- $8-C-\beta-D$-glucoside \\
\hline 9 & 6.4 & 222,388 & $\begin{array}{l}\mathrm{MS}^{2}[577]: 457(100.0), 559(8.2), 517(1.3), 487(45.4), \\
367(21.1), 337(39.8)\end{array}$ & Chrysin-6,8-C-diglucoside \\
\hline 10 & 6.7 & $224,272,316$ & $\begin{array}{l}\mathrm{MS}^{2}[547]: 487(100.0), 529(14.5), 457(79.3), 427(79.3) \text {, } \\
367(41.0), 337(85.9)\end{array}$ & Chrysin-6-C- $\alpha$-L-arabinoside-8-C- $\beta$-D-glucoside \\
\hline 11 & 7.1 & 269,316 & $\begin{array}{l}\mathrm{MS}^{2}[547]: \text { 457(100.0), 529(22.4), 487(55.3), } 427 \text { (58.9), } \\
367(48.2), 337(50.3)\end{array}$ & $\begin{array}{l}\text { Chrysin-3-C- } \alpha \text {-arabinopyranosyl- } 8-C-\beta \text { - } \\
\text { glucopyranoside }\end{array}$ \\
\hline 12 & 8.6 & 267,313 & $\mathrm{MS}^{2}[511]: 431(100.0), 349(10.6), 269(3.2), 241(1.2)$ & 6-Sulfooxy-chrysin 7-glucoside \\
\hline 13 & 10.1 & $220,274,316$ & $\begin{array}{l}\operatorname{MS}^{2}[547]: 457(100.0), 529(10.4), 487(38.1), 427(22.6), \\
367(34.6)\end{array}$ & Chrysin-6-C- $\beta$-D-glucoside- $8-C-\alpha L$-arabinoside \\
\hline 14 & 10.6 & 279,317 & $\mathrm{MS}^{2}[511]: 269(100.0), 431(20.5), 351(4.2), 349(3.1), 241(8.6)$ & Bicalein-7-O- $\beta$-D-glucopyranosyl sulfate conjugate \\
\hline 15 & 11.6 & 252,347 & $\mathrm{MS}^{2}[475]: 299(100.0), 300(26.2), 284(5.4), 175(3.7)$ & Chrysoeriol-7-O- $\beta$-D-glucuronide \\
\hline 16 & 13.6 & 276,315 & $\mathrm{MS}^{2}[431]: 269(100.0), 271(3.3), 270(16.5), 201(1.2), 196(1.0)$ & Baicalein-7-O- $\beta$-D-glucopyranoside \\
\hline 17 & 14.0 & 272,316 & $\mathrm{MS}^{2}[445]:$ 430(100.0), 283(87.6), 268(82.4), 161 (1.2) & Wogonin-5-O- $\beta$-D-glucopyranoside \\
\hline 18 & 14.7 & 270,313 & $\mathrm{MS}^{2}[415]: 295(100.0), 325(17.2), 296(14.2), 267(3.2)$ & Chrysin-8-C- $\beta$-D-glucopyranoside \\
\hline 19 & 16.4 & 276,315 & $\begin{array}{l}\mathrm{MS}^{2}[445]: \text { 269(100.0), 366(2.5), 341(2.2), 270(19.5), } \\
223(3.3), 175(20.7)\end{array}$ & Baicalein-7-O-glucuronide (baicalin) \\
\hline 20 & 16.6 & 263,323 & $\begin{array}{l}\mathrm{MS}^{2}[475]: \text { 299(100.0), 301(3.2), 300(19.4), 285(2.2), } \\
\text { 284(37.1), 175(5.2) }\end{array}$ & $\begin{array}{l}5,7,6^{\prime} \text {-trihydroxy-2'-methoxy flavone-7-O- } \beta \text {-D- } \\
\text { glucuronide }\end{array}$ \\
\hline 21 & 17.4 & 282,333 & $\mathrm{MS}^{2}[445]: 269(100.0), 427(2.0), 385(1.0), 175(14.9), 137(2.9)$ & $\begin{array}{l}\text { Apigenin-7-O- } \beta \text {-D-glucuronopyranoside } \\
\text { (scutellarin A) }\end{array}$ \\
\hline 22 & 17.7 & 268,336 & $\mathrm{MS}^{2}[445]: 269(100.0), 244(35.0), 216(1.4), 175(2.6)$ & 2'-Hydroxychrysin-7-glucuronide \\
\hline 23 & 18.1 & 254,346 & $\begin{array}{l}\mathrm{MS}^{2}[463]: 161(100.0), 301(68.4), 283(3.4), 191(8.1), \\
162(12.7), 139(5.5)\end{array}$ & $\begin{array}{l}\text { Quercetin-7-O- } \beta \text {-D-glucopyranoside } \\
\text { (quercimeritrin) }\end{array}$ \\
\hline 24 & 18.4 & 287,340 & $\mathrm{MS}^{2}$ [409]: 329(100.0), 315(0.2), 314(0.9), 299(0.2) & 5-Hydroxy-6,7,4'-trimethoxy flavanone-5-sulfate \\
\hline 25 & 18.9 & 272,312 & $\operatorname{MS}^{2}[459]: 283(100.0), 268(8.6), 175(20.7)$ & Oroxylin A-7-O- $\beta$-D-glucuronide \\
\hline 26 & 19.3 & 274,349 (sh) & $\mathrm{MS}^{2}[459]: 283(100.0), 269(5.0), 268(4.2), 176(1.3), 175(24.5)$ & Wogonin-7-O- $\beta$-D-glucuronopyranoside \\
\hline 27 & 21.5 & 276,320 & $\mathrm{MS}^{2}[349]: 269(100.0), 332(0.1), 270(23.0), 225(0.3)$ & Baicalein-7-O-sulfate \\
\hline 28 & 23.1 & 288 & $\begin{array}{l}\mathrm{MS}^{2}[303]: 125(100.0), 285(3.1), 275(9.0), 259(8.7), \\
217(25.3), 177(30.9)\end{array}$ & 3,5,7,3',4'-pentahydroxy flavanone (taxifolin) \\
\hline 29 & 24.4 & 324,287 & $\mathrm{MS}^{2}[303]: 125(100.0), 285(6.1), 259(15.6), 177$ (12.9) & (2R,3R)-3,5,7,2',6'-pentahydroxy flavanone \\
\hline 30 & 26.4 & 290,330 & $\begin{array}{l}\mathrm{MS}^{2}[303]: 125(100.0), 285(4.4), 275(10.2), 259(6.1), \\
217(13.8), 177(27.6)\end{array}$ & $3,5,7,2^{\prime}, 6$ '-Pentahydroxy flavanone \\
\hline 31 & 27.6 & 254,351 & $\mathrm{MS}^{2}[301]: 161(100.0), 283(4.8), 273(3.8), 270(1.2), 139(44.0)$ & ,'-Penta \\
\hline 32 & 28.1 & 253,302 & $\begin{array}{l}\mathrm{MS}^{2}[301]: 161(100.0), 283(7.5), 273(17.9), 269(18.2), \\
139(86.4), 133(15.3)\end{array}$ & $5,7,2^{\prime}, 6^{\prime}$-Tetrahydroxy flavonol (visciclulin I) \\
\hline 33 & 31.1 & 267,342 & $\operatorname{MS}^{2}[345]: 330(100.0), 331(17.5), 329(4.9), 328(1.4), 315(5.1)$ & $\begin{array}{l}5,7,2^{\prime}, 5^{\prime} \text {-Tetrahydroxy-8,6'-dimethoxy flavone } \\
\text { (viscidulin III) }\end{array}$ \\
\hline 34 & 31.5 & 261,310 & $\begin{array}{l}\mathrm{MS}^{2}[285]: \text { 125(100.0), 269(5.6), 241(1.0), 177(1.0), } \\
\text { 161(28.7), 151(2.6), }\end{array}$ & $5,7,2^{\prime}, 6^{\prime}$-Tetrahydroxy flavone \\
\hline 35 & 34.2 & 275,340 & $\mathrm{MS}^{2}[393]: 313(100.0), 331(0.9), 314(8.1), 298(0.7)$ & 5,2'-Dihydroxy-7,8-dimethoxy flavone (panicolin) \\
\hline 36 & 35.2 & 272,313 & $\mathrm{MS}^{2}[283]: 269(100.0), 175(4.1)$ & 5,7-Dihydroxy-6-methoxy flavone (oroxylin A) \\
\hline 37 & 36.3 & 262,346 & $\mathrm{MS}^{2}[285]: 241(11.0), 237(7.6), 227(9.9), 151(10.0)$ & $3,5,7,4^{\prime}$-Tetrahydroxy flavone (kaempferol) \\
\hline 38 & 36.8 & $241,253,349$ & $\begin{array}{l}\mathrm{MS}^{2}[285]: 241(6.8), 233(5.8), 231(2.4), 201(6.7), 199(1.2), \\
175(4.9), 151(13.7)\end{array}$ & $5,7,3^{\prime}, 4^{\prime}$-Tetrahydroxy flavone (luteolin) \\
\hline 39 & 37.9 & $222,278,304$ & $\begin{array}{l}\mathrm{MS}^{2}[285]: 267(10.0), 243(2.5), 223(3.5), 218(4.1), 201(9.1), \\
153(5.3), 133(9.1)\end{array}$ & 5,7,8,4'-tetrahydroxy flavone (isoscutellarein) \\
\hline 40 & 48.5 & 276,320 & $\mathrm{MS}^{2}[285]: 268(18.5), 241(7.2), 213(18.7), 205(3.4), 149(1.3)$ & $5,6,7,4^{\prime}$-Tetrahydroxy fla \\
\hline 41 & 49.6 & $260(\mathrm{sh}), 274,362$ & $\operatorname{MS}^{2}[331]: 331(100.0), 300(1.2), 273(10.7), 157(1.8)$ & $5,7,3^{\prime}, 4^{\prime}, 5^{\prime}$-Pentahydroxy-3,6,8-trimethoxy flavone \\
\hline 42 & 50.4 & 274 & $\mathrm{MS}^{2}[283]: 268(100.0), 283(4.2), 269(16.2), 137(5.4)$ & 5,7-Dihydroxy-8-methoxy flavone (wogonin) \\
\hline 43 & 51.0 & $230,253,311(\mathrm{sh})$ & $\mathrm{MS}^{2}[253]: 181(100.0), 208(9.4), 164(20.9), 125(47.7)$ & 7,4'-Dihydroxy flavone \\
\hline 44 & 51.1 & 268,315 & $\mathrm{MS}^{2}[253]: 153(100.0), 186(4.5), 171(3.9), 145(13.7)$ & 5,7-Dihydroxy flavone (chrysin) \\
\hline 45 & 51.2 & 269,324 & $\mathrm{MS}^{2}[283]: 267(100.0), 136(1.8)$ & 5,7-Dihydroxy-4'-methoxy flavone (acacetin) \\
\hline
\end{tabular}






1

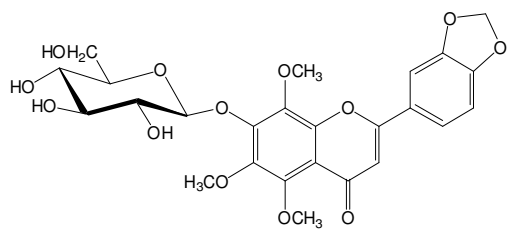

2

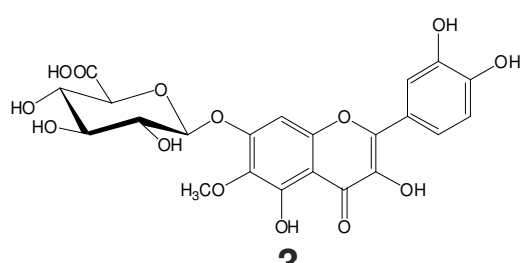

3



4

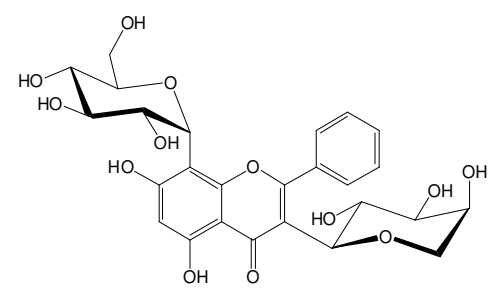

11



12

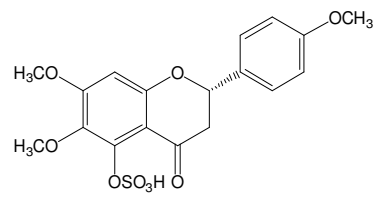

24

Fig. 2. Structures of the new components in the extract of S. baicalensis

12.0, $8.8 \mathrm{~Hz}, \mathrm{H}-3), 2.81(1 \mathrm{H}, \mathrm{dd}, J=12.0,3.8 \mathrm{~Hz}, \mathrm{H}-3) ;{ }^{13} \mathrm{C}$ NMR (DMSO- $\left.d_{6}, 100 \mathrm{MHz}\right) \delta 197.5(\mathrm{C}-4), 159.7\left(\mathrm{C}-4{ }^{\prime}\right), 158.6$ (C-7), 155.7 (C-5), 153.5 (C-9), 133.6 (C-6), 130.3 (C-1'), 128.4 (C-2', C-6'), 113.8 (C-3', C-5'), 106.2 (C-10), 90.5 (C8), $78.9(\mathrm{C}-2), 59.9\left(7-\mathrm{OCH}_{3}\right), 58.2\left(6-\mathrm{OCH}_{3}\right), 55.1\left(4 '-\mathrm{OCH}_{3}\right)$, 42.7 (C-3); ESI-MS/MS data (Table-1).

Supporting information: NMR spectra $\left({ }^{1} \mathrm{H}\right.$ NMR, ${ }^{13} \mathrm{C}$ NMR, HMQC and HMBC), HR-ESI-MS of all new compounds and detailed extrction and isolation procedures are given as supporting information.

\section{RESULTS AND DISCUSSION}

Systematic phytochemical investigations were carried out to probe the chemical profile of the S. baicalensis, the successive purification of the EtOH extract of $S$. baicalensis by medium-pressure ODS column chromatography. Seven new and 38 known compounds were isolated and purified through medium-pressure ODS column, as well as repeated preparative using semi-preparative HPLC.

Compound 1, obtained as pale yellow needles crystals. The molecular formula of $\mathbf{1}$ was determined to be $\mathrm{C}_{25} \mathrm{H}_{26} \mathrm{O}_{13}$ on the basis of negative ion ESI-MS, ${ }^{1} \mathrm{H}$ and ${ }^{13} \mathrm{C}$ NMR spectral data. In the UV spectrum of $\mathbf{1}$, the maximum bands are at 256 , 273 and $350 \mathrm{~nm}$. Its IR spectrum showed absorption bands due to hydroxy $\left(3100 \mathrm{~cm}^{-1}\right)$, carbonyl $\left(1620 \mathrm{~cm}^{-1}\right)$, aromatic $\left(1605,1590,1495 \mathrm{~cm}^{-1}\right)$ groups. In the ${ }^{1} \mathrm{H}$ NMR spectrum (Table-1), three singlets ( $3 \mathrm{H}$, each) were observed at $\delta_{\mathrm{H}}=3.72$, 3.75 and 3.81, indicating three methoxyl groups. The ${ }^{13} \mathrm{C} N M R$ signals of 1 were assigned with the help of an HMQC experiment, establishing direct $\mathrm{C}-\mathrm{H}$ bonding. The location of the three methoxyl groups were assigned to C-3, C-5 and C-8 based on the observed $\mathrm{HMBC}$ correlations from $3-\mathrm{OCH}_{3}(\delta 3.72)$, $5-\mathrm{OCH}_{3}(\delta 3.81)$ and $8-\mathrm{OCH}_{3}(\delta 3.75)$ to $\mathrm{C}-3(\delta 140.9), \mathrm{C}-5(\delta$ $157.1)$ and $\mathrm{C}-8(\delta 131.0)$, respectively. This experiment also clarified the site of glycosidation showing a long-range correlation between the anomeric proton of glucose at $\delta_{\mathrm{H}} 5.11(\mathrm{~d}$, $J=7.7 \mathrm{~Hz}, \mathrm{H}-1 "$ ") and the oxygenated carbon atom at $\delta_{\mathrm{C}} 155.4$ (C-7). The ${ }^{1} \mathrm{H}$ and ${ }^{13} \mathrm{C}$ data of the aglycone were similar to those reported for 7-hydroxy-3,5,8-trimethoxy-3',4'-methylenedioxyflavone ${ }^{4}$. Finally, the structure of 1 was established as 7-hydroxy-3,5,8-trimethoxy-3',4'-methylenedioxy flavone7-O- $\beta$-glucopyranoside.
Compound 2, obtained as pale yellow needles with the molecular formula $\mathrm{C}_{25} \mathrm{H}_{26} \mathrm{O}_{13}$ (negative ion ESI-MS, ${ }^{1} \mathrm{H}$ and ${ }^{13} \mathrm{C}$ NMR spectral data). The ${ }^{1} \mathrm{H}$ and ${ }^{13} \mathrm{C}$ NMR spectra of 2 were similar to those of $\mathbf{1}$, which are different with site of methoxyl linkage. Comparison with the spectrum of compound 1 allowed an upfield shift for C-3 signal and a downfield shift for the C-6 signal, furthermore, signals of methoxyl groups could be assigned to C- 6 . The structure of aglycone was identified by comparison of its MS and ${ }^{1} \mathrm{H}$ and ${ }^{13} \mathrm{C}$ NMR data with those reported in the literature 5 . Based on these findings, the chemical structrue of $\mathbf{2}$ has been elucidated to be 7-hydroxy5,6,8-trimethoxy-3',4'-methylenedioxy flavone-7-O- $\beta$-glucopyranoside.

Compound $\mathbf{3}$, the ${ }^{13} \mathrm{C}$ NMR spectrum of $\mathbf{3}$ displayed the presence of one glucuronide unit in addition to 16 carbon signals for the aglycone. The chemical shifts of the aglycone carbons were similar to those reported for patuletin ${ }^{6}$. In the HMBC spectrum, long-rang correlations were observed from $\delta 5.17$ (H-1 of GlcA) to $\delta 157.3$ (C-7 of the aglycone). Accordingly, the structure of 3 was formulated as patuletin 7- $\beta$-glucuronide.

Compound 4 is optically active, $[\alpha]_{D}^{25}=10.1$ (c 0.5, $\mathrm{MeOH}$ ) and its molecular formula was established as $\mathrm{C}_{26} \mathrm{H}_{30} \mathrm{O}_{14}$ by high resolution ESIMS. Additionally, two anomeric proton resonances were observed at $\delta 4.64(\mathrm{~d}, J=9.0 \mathrm{~Hz})$ and $4.80(\mathrm{~d}, J=9.6 \mathrm{~Hz})$, indicating its diglycosidic structure. The sugar functionality was identified as a $\beta$-glucopyranose and arabinopyranoside by ${ }^{1} \mathrm{H}$ and ${ }^{13} \mathrm{C}$ NMR spectral data together with ESI-MS. The ${ }^{13} \mathrm{C}$ NMR spectrum of $\mathbf{4}$ exhibited 26 carbon resonances: 8 quaternary carbons $(\mathrm{C}), 16$ methine $(\mathrm{CH})$ and 2 methylene $\left(\mathrm{CH}_{2}\right)$. In the HMBC spectrum, the proton signal at $\delta 4.51(\mathrm{~d}, J=12.8 \mathrm{~Hz}, \mathrm{H}-2)$ showed long-range correlations with the carbon signals at $\delta 160.5$ (C-9), whereas the anomeric proton $(\delta 4.64)$ showed long-range correlation with C-8 $(\delta 105.4)$ and C-9 $(\delta 160.5)$, indicating that the arabinose moiety was located at $\mathrm{C}-8$. In the HMBC spectrum, the longrang correlation between the anomeric proton and $\mathrm{C}-3$ " $(\delta 77.7)$ of the glucose moiety, correlation to C-5 ( $\delta$ 161.5)/C-6 ( $\delta$ $106.9) / C-7$ ( $\delta 165.1)$, confirmed the attachment of glucose moiety to the carbon C-6. The structures of aglycone were identified by comparison of its MS and ${ }^{1} \mathrm{H}$ and ${ }^{13} \mathrm{C}$ NMR data with those reported in the literature ${ }^{7}$. Therefore, the structure 
of 4 can be identified as pinobankasin-6-C-glucopyranosyl-

8-C-arabinopyranoside.

Compound 11, the ${ }^{1} \mathrm{H}$ and ${ }^{13} \mathrm{C}$ NMR spectral data together with ESI-MS supported the presence of diglycosidic. The structure of the sugar moieties could be entirely established on the basis of ${ }^{1} \mathrm{H}$ and ${ }^{13} \mathrm{C}$ NMR data. In $\mathrm{HMBC}$, the anomeric proton $(\delta 4.64)$ showed long-range correlation with C-8 ( $\delta 105.1), \mathrm{C}-$ $9(\delta 154.3)$ and $C-3 "(\delta 79.1)$ indicating that the glucose moiety was located at C-8. Moreover, this experiment also clarified the site of glycosidation showing a long-range correlation between the anomeric proton of arabinose at $\delta_{\mathrm{H}} 4.80(\mathrm{~d}, J=$ $9.4 \mathrm{~Hz})$ and the carbon atom at $\delta_{\mathrm{C}} 178.3(\mathrm{C}-4)$. Thus, compound 11 was identified as chrysin $3-\mathrm{C}-\alpha$-arabinopyranosyl8 -C- $\beta$-glucopyranoside by comparing its ${ }^{1} \mathrm{H}$ and ${ }^{13} \mathrm{C}$ NMR data with previously published data ${ }^{8}$.

Compound 12, obtained as a yellow amorphous powder. The molecular formula was determined to be $\mathrm{C}_{21} \mathrm{H}_{20} \mathrm{O}_{1} 3 \mathrm{~S}$ on the basis of negative ion ESI-MS, ${ }^{1} \mathrm{H}$ and ${ }^{13} \mathrm{C}$ NMR spectral data. The UV spectrum exhibited maxima at 267 and $313 \mathrm{~nm}$ and IR spectrum suggested the presence of hydroxy groups $\left(3348 \mathrm{~cm}^{-1}\right)$. The ${ }^{1} \mathrm{H}$ and ${ }^{13} \mathrm{C}$ data of the aglycone were similar to those reported for chrysin. The significant shifts of the carbon signals of the ipso, ortho and para positions caused by the sulphation at C-6 are consistent with literature data for flavonoid sulphates ${ }^{9}$. The $\beta$-glucopyranosyl unit is linked to C-7 of the aglycone from the chemical shift of this carbon at $\delta$ 166.5. Therefore, the structures of compound 12, 6-sulfoxychrysin-7-glucoside, were identified on the basis of comparison of its spectroscopic (NMR, MS) data in comparison with literature values ${ }^{10}$.

Compound $\mathbf{2 4}$ was isolated as an pale yellow crystal. The molecular formula was established as $\mathrm{C}_{18} \mathrm{H}_{18} \mathrm{O}_{9} \mathrm{~S}$ by ESI-MS, ${ }^{1} \mathrm{H}$ and ${ }^{13} \mathrm{C}$ NMR spectral data. The IR spectrum showed absorption bands at $1630(\mathrm{C}=\mathrm{O}), 1445$ (aromatic), 1270 (-C-O-) and the UV spectrum exhibited maxima at 225, 287 and 340 $\mathrm{nm}$. The signal at $\delta 6.71$ related to two methylene protons showed long-range correlations with the signals for C-7, C-9, C-6 and C-10, whereas the methoxy protons at $\delta 3.80$ and $\delta$ 3.85 showed long-range correlation with C-5 and C-6, repectively. Additional, the signal at $\delta_{\mathrm{H}} 3.78$ showed long-range correlations with the signals for C-3', C-5' and 4'- $\mathrm{OCH}_{3}$ in the $\mathrm{B}$ ring. The significant shifts of the carbon signals of the ipso, ortho and para positions caused by the sulphation at C-5. Therefore, indicating the sulphation at C-5. Based on these findings, compound $\mathbf{2 5}$ were identified by comparing their physical and spectral data with the literature values: 5-hydroxy6,7,4'- trimethoxy flavanone 5-sulfate ${ }^{11}$.

By comparing their physical and spectroscopic data with published data, the structures of the other 38 known chemical constituents were elucidated as 6,8-dihydroxy quercetin diglucopyranoside $(\mathbf{5})^{12}, 3,5,7,8,3^{\prime}, 4^{\prime}$-hexahydroxy-6-methoxy flavone diglucopyranoside $(6)^{13}$, apigenin-6-C- $\beta$-D-glucoside8-C- $\alpha$-L-arabinoside $(7)^{14}$, apigenin-6-C- $\alpha$-L-arabinoside- 8 C- $\beta$-D-glucoside $(\mathbf{8})^{14}$, chrysin-6,8-C-diglucoside $(9)^{15}$, chrysin-6-C- $\alpha$-L-arabinoside- $8-C-\beta$-D-glucoside $(10)^{16}$, chrysin-6-C- $\beta$-D-glucoside- 8 -C- $\alpha$-L-arabinoside $(\mathbf{1 3})^{16}$, baicalein-7-O- $\beta$-D-glucopyranosyl sulfate conjugate $(\mathbf{1 4})^{17}$, chrysoeriol-7-O- $\beta$-D-glucuronide $(\mathbf{1 5})^{18}$, baicalein-7-O- $\beta$-D- glucopyranoside $(\mathbf{1 6})^{17}$, wogonin-5-O- $\beta$-D-glucopyranoside $(\mathbf{1 7})^{19}$, chrysin-8-C- $\beta$-D-glucopyranoside $(\mathbf{1 8})^{20}$, baicalein-7O-glucuronide (baicalin) (19) $)^{21}, 5,7,6$ '-trihydroxy-2'-methoxy flavone-7-O- $\beta$-D-glucuronide $(\mathbf{2 0})^{22}$, apigenin 7-O- $\beta$-Dglucuronopyranoside (scutellarin A) $(\mathbf{2 1})^{23}, 2^{\prime}$-hydroxychrysin7-glucuronide $(\mathbf{2 2})^{24}$, quercetin-7-O- $\beta$-D-glucopyranoside (quercimeritrin) $(\mathbf{2 3})^{25}$, oroxylin A-7-O- $\beta$-D-glucuronide $(\mathbf{2 5})^{26}$, wogonin-7-O- $\beta$-D-glucuronopyranoside (26), baicalein7-O-sulfate $(27)^{27}, 3,5,7,3^{\prime}, 4^{\prime}$-pentahydroxy flavanone (taxifolin) $(\mathbf{2 8})^{28},(2 \mathrm{R}, 3 \mathrm{R})-3,5,7,2^{\prime}, 6^{\prime}$-pentahydroxy flavanone $(\mathbf{2 9})^{29}$, $3,5,7,2^{\prime}, 6^{\prime}$-pentahydroxy flavanone $(\mathbf{3 0})^{30}, 3,5,7,3^{\prime}, 4^{\prime}$ '-pentahydroxy flavone (quercetin) (31), 5,7,2',6'-tetrahydroxy flavonol (visciclulin I) $(32)^{31}, 5,7,2^{\prime}, 5^{\prime}$-tetrahydroxy-8,6'dimethoxy flavone (viscidulin III) (33 $)^{32}, 5,7,2^{\prime}, 6^{\prime}$-tetrahydroxy flavone $(\mathbf{3 4})^{33}, 5,2^{\prime}$-dihydroxy-7,8-dimethoxy flavone (panicolin) $(35)^{34}, 5,7$-dihydroxy-6-methoxy flavone (oroxylin A) $(\mathbf{3 6})^{35}, 3,5,7,4^{\prime}$-tetrahydroxy flavone (kaempferol) $(37)^{36}$, $5,7,3^{\prime}, 4^{\prime}$-tetrahydroxy flavone (luteolin) $(\mathbf{3 8})^{37}, 5,7,8,4^{\prime}$ tetrahydroxy flavone (isoscutellarein) $(\mathbf{3 9})^{38}, 5,6,7,4^{\prime}-$ tetrahydroxy flavone (scutellarein) $(\mathbf{4 0})^{36}, 5,7,3^{\prime}, 4^{\prime}, 5^{\prime}-$ pentahydroxy-3,6,8-trimethoxy flavone $(\mathbf{4 1})^{39}, 5,7$-dihydroxy8-methoxy flavone (wogonin) (42) ${ }^{40}, 7,4$ '-dihydroxy flavone $(\mathbf{4 3})^{41}, 5,7$-dihydroxy flavone (chrysin) $(\mathbf{4 4})^{42}, 5,7$-dihydroxy4'-methoxy flavone (acacetin) $(\mathbf{4 5})^{43}$.

\section{ACKNOWLEDGEMENTS}

The authors thank the National Science and Technology Major Project Key New Drug Creation and Manufacturing Program, China (No. 2009ZX09301-001, 2009ZX09308-005) and the National Natural Science Foundation of China (No. 81030065) for financial support of this work.

\section{REFERENCES}

1. National Commission of Chinese Pharmacopoeia, Pharmacopoeia of the People's Republic of China. China Medical Technology Press, Beiing (2010).

2. Jiangsu New Medical College, Dictionary of Chinese Materia Medical, Science and Technology Press of ShangHai (1977).

3. H.Z. Wen, S.Y. Xiao, Y.M. Wang and G.A. Luo, Nat. Prod. Res. Dev., 6, 575 (2004).

4. G. Corinne, M. Frederic, B. Francoise, T. Francois, V. Jacqueline, S. Thierry and L. Marc, J. Nat. Prod., 62, 1188 (1999).

5. W.L. Yang, H.F. Chen, B.J. Yu, P.Z. Zhang, Z.H. Yan, L. Jie and H. Xu, Zhong Yao Cai, 31, 1812 (2008).

6. H. Tokunaru, S. Kenichi, Y. Kazuyo, K. Yasuhiko and T. Masao, Chem. Pharm. Bull., 45, 446 (1997).

7. K. Masanori, Y. Yooko, F. Seigo, U. Akira, N. Tadataka and M. Toshio, Chem. Pharm. Bull., 30, 1602 (1982).

8. D.W. Aksnes, A. Standnes and O.M. Anderson, Magn. Reson. Chem., 34, 820 (1996).

9. H.J. Xia, F. Qiu, S. Zhu, T.Y. Zhang, G.X. Qu and X.S. Yao, Biol. Pharm. Bull., 30, 1308 (2007).

10. E.A. Ali, M. Ibtissam, T. Rachida Chendid, B. Mohamed, E.A. Rachid, E.B. Brahim and L. Mohammed, Molecules, 9, 568 (2004).

11. E. Wollenweber, V.H. Dietz, D. Schillo and G. Schilling, Zeitsch. Naturforschung, C: J. Biosci., 35, 685 (1980).

12. D.K. Bhardwaj and R.M. Jain, Proc. Indian Nat. Sci. Acad., 49, 408 (1983).

13. L.C. Dos Santos, C.M. Rodrigues, M.A. Silva, R.G. Coelho, M. Sannomiya and W. Vilegas, Biochem. System. Ecol., 33, 1159 (2005).

14. C. Xie, N.C. Veitch, P.J. Houghton and S.J. Simmonds, Chem. Pharm. Bull., 51, 1204 (2003).

15. L. Gobbo-Neto, M.D. Santos, A. Kanashiro, M.C. Almeida, Y.M. Lucisano-Valim, L.C. Lopes, E.P. Souza and N.P. Lopes, Planta Medica, 71, 3 (2005). 
16. S. Takagi, M. Yamaki and K. Inoue, Yakugaku Zasshi, 101, 899 (1981).

17. Y. Yuan, W.L. Hou, M.H. Tang, H.D. Luo, L.J. Chen, Y.H. Guan and L.A. Sutherland, Chromatographia, 68, 885 (2008).

18. G. Romussi, N. Fontana and N.D. Tommasi, Phytother. Res., 10, S84 (1996).

19. Y.Y. Zhang, Y.Z. Guo, H. Ageta, Y. Harigaya, M. Onda, K. Hashimoto, Y. Ikeya, M. Okada and M. Maruno, J. Chin. Pharm. Sci., 6, 182 (1997).

20. Z.H. Zhou and C.R. Yang, Yunnan Zhiwu Yanjiu, 22, 475 (2000).

21. T. Tomimori, Y. Miyaichi, Y. Imoto, H. Kizu and Y. Tanabe, Yakugaku Zasshi, 103, 607 (1983).

22. J. Han, M. Ye, M. Xu, J.H. Sun, B.R. Wang and D. Guo, J. Chromatogr. B, 848, 355 (2007)

23. T .Hase, K. Ohtani, R. Kasai, K. Yamasaki and C. Picheansoonthon, Phytochemistry, 40, 287 (1995).

24. Y.Q. Wang, K. Matsuzaki, K. Takahashi, T. Okuyama and S. Shibata, Chem. Pharmaceut. Bull., 36, 3206 (1988).

25. R. Kunde and O. Isaac, Planta Medica, 37, 124 (1979).

26. K. Abe, O. Inoue and E. Yumioka, Chem. Pharm. Bull., 38, 208 (1990).

27. W.H. Huang, A.R. Lee and C.H. Yang, Biosci. Biotechnol. Biochem., 70, 2371 (2006)

28. W.L. Mei, W. Ni, Y. Hua and C.X. Chen, Nat. Prod. Res. Dev., 14, 26 (2002).

29. Y.Y. Zhang and Y.Z. Guo, Shenyang Yaoxueyuan Xuebao, 8, 137 (1991).

30. S. Takagi, M. Yamaki and K. Inoue, Phytochemistry, 20, 2443 (1981).
31. H.Y. Wang, L.H. Xiao, L. Liu and S.X. Xu, Shenyang Yaoke Daxue Хиebao, 20, 339, 348 (2003).

32. M.L. Liu, M.L. Li, X.T. Liang, X.Y. Lin, Q.T. Zheng, S.D. Zhang and F.L. Shen, Zhongcaoyao, 17, 438 (1986).

33. T. Tomimori, Y. Miyaichi and H. Kizu, Yakugaku Zasshi, 102, 388 (1982).

34. M. Inuma, S. Matsuura and K. Kusuda, Chem. Pharm. Bull., 28, 708 (1980).

35. R.R. Biekofsky, C.A. Buschi and A.B. Pomilio, Magn Reson. Chem., 29, 569 (1991).

36. L.H. Qin, X.Y. Guo, M. Fan, A.S. Din, N.L. Wang and X.S. Yao, Shenyang Yaoke Daxue Xuebao, 23, 633 (2006).

37. J.Y. Peng, G.R. Fan and Y.T. Wu, China J .Chin. Mater. Medica, 31, 128 (2006).

38. W.H. Huang, Y.B. Li and J.Q. Jiang, Zhongguo Zhongyao Zazhi, 34, $712(2009)$

39. S.G. Yu, N.B. Fang and T.J. Mabry, Phytochemistry, 27, 171 (1988).

40. X. Li, J.H. Wang, X. Li and D.L. Liang, Shenyang Yaoke Daxue Xuebao, 19, 178 (2002).

41. C.N. He, W.W. Gao, W.Y. Xu and J.M. Tong, Zhong Cao Yao, 39, 1783 (2008).

42. Z.T. Ma and X.W. Yang, Zhongguo Zhongyao Zazhi, 33, 2080 (2008).

43. N.Y.Yang, J.H. Duang, P. Li, S.H. Qian and W.C. Hu, Zhongguo Tianran Yaowu, 4, 98 (2006). 